\title{
Enhanced Turbulence and Heating Observed Preceding the Impulsive Phase in a Solar Flare
}

\author{
Chung-Chieh Cheng and K.G. Widing \\ E.O. Hulburt Center for Space Research \\ Naval Research Laboratory \\ Washington, D.C. 20375-5000. U.S.A.
}

\begin{abstract}
We studied the unique EUV spectra of the $21 \mathrm{Jan} .1974$ flare observed by the NRL Normal-Incidence Slit Spectrometer (SO 82B) onboard the Skylab. The results show that the pre-impulsive transition region plasmas exhibited enhanced turbulence and heating before the acceleration of energetic particles. The absence of blue-shifted components in the Fe XXI line profiles shows that the chromospheric evaporation is not important in this flare.
\end{abstract}

\section{INTRODUCTION}

To understand the energy build-up during the evolution of the active region that leads to the flare energy release and particle acceleration, it is necessary to know the physical conditions of the preflare state. The preflare state is generally referred to as the time period before the onset of the impulsive phase during which impulsive hard X-ray (HXR) and UV bursts are emitted. Although numerous observations in various wavelength bands ranging from radio, optical, to X-rays exist, the preflare state is still poorly understood.

Tn this paper, we studied the unique UV spectra of the 21 January 1974 flare obtained from Skylab. The UV spectra of the flare were taken a few minutes before and during the impulsive phase of the flare, thus affording us a rare opportunity of investigating the physical conditions that prevail in the chromosphere and transition region prior to the acceleration of energetic particles in the impending impulsive phase. Previous analyses of the UV line jirofiles from flares have been limited to the post-impulsive phase (Cheng 1978. Doschek, Feldman, and Rosenberg 1977, Dere and Cook 1979).

\section{OBSERVATIONS}

The UV spectra of the 21 January flare were obtained with the NRL slit normalincidence grating spectrograph (SO 82B) onboard Skylab. The spectrograph。 recorded solar radiation in the wavelength range $940-1940 \AA$ or $1940-3940 \AA$, with a spectral resolution of $0.06 \AA$. The projected area on the Sun was $2^{n} x$ $60^{n}\left(1450 \times 43500 \mathrm{~km}^{2}\right)$. A complete description of the instrument $\mathrm{can}$ be found in Bartoe et al. (1977). For details of the methodology used in reducing the 82B spectra, see Cheng (1978).

The 21 January 1974 flare was a SN flare winch occurred in McMath region 12708 near the west limb. This flare was also observed by the NRL XUV slitless spectrograph (SO 82A) in the wavelengch region from 170 to $640 \AA$. The XUV imaging observations of this flare have been analyzed by Brueckner (1977), Cheng (1977), and Widing and Iliei (1984). The XUV images show that the flare m:orphology consisted of two interacting loops; one large loop. best observed in Fe II, and a smaller loop in which the high temperature flare Fe XXIV plasma 
was contained. The $82 \mathrm{~B}$ slit was pointed at the common footpoint of the interacting loops and also covered part of the Fe XXIV plasma as shown schematically in Fig. 1. The first UV spectrum was taken at 2313 UT, about 3 minutes before the impulsive HXR burst; see Fig. 2 .

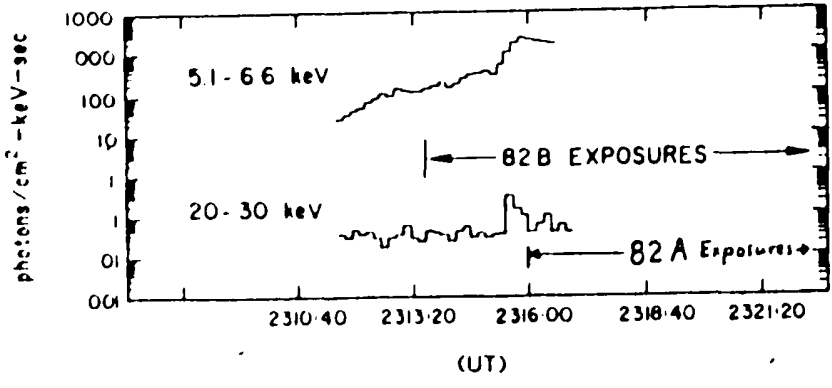

Fig. 1

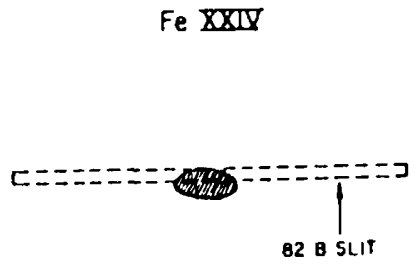

He II LOOP

Fig. 2

\section{Line Profile Analysis}

Examination of the UV spectra taken before and during the impulsive phase shows that all of the transition region lines had broadened line profiles. We fitted the observed line profiles with two Gaussian components. In deriving the absolute intensity of the emission lines, the continuum level has been sultracted. Figures 3,4 , and 5 show the observed profiles of the $N \mathrm{~V}$ ( 1238 $\AA), O V(1371 \AA)$, and Si IV (1402 $⿱$ ) lines, and the corresponding fitted profiles with two Gaussian components. As can be seen from the figures, the two-component Gaussian fits rather well. It is clear that the observed line profiles consisted of sharp and broad components with no relative line shifts between them. The sharp components were emissions from the background active region, and the broad components were from the flaring kernel. The nonthermal mass motion velocity for the sharp components ranges from 20 to $40 \mathrm{~km} \mathrm{~s}^{-1}$.
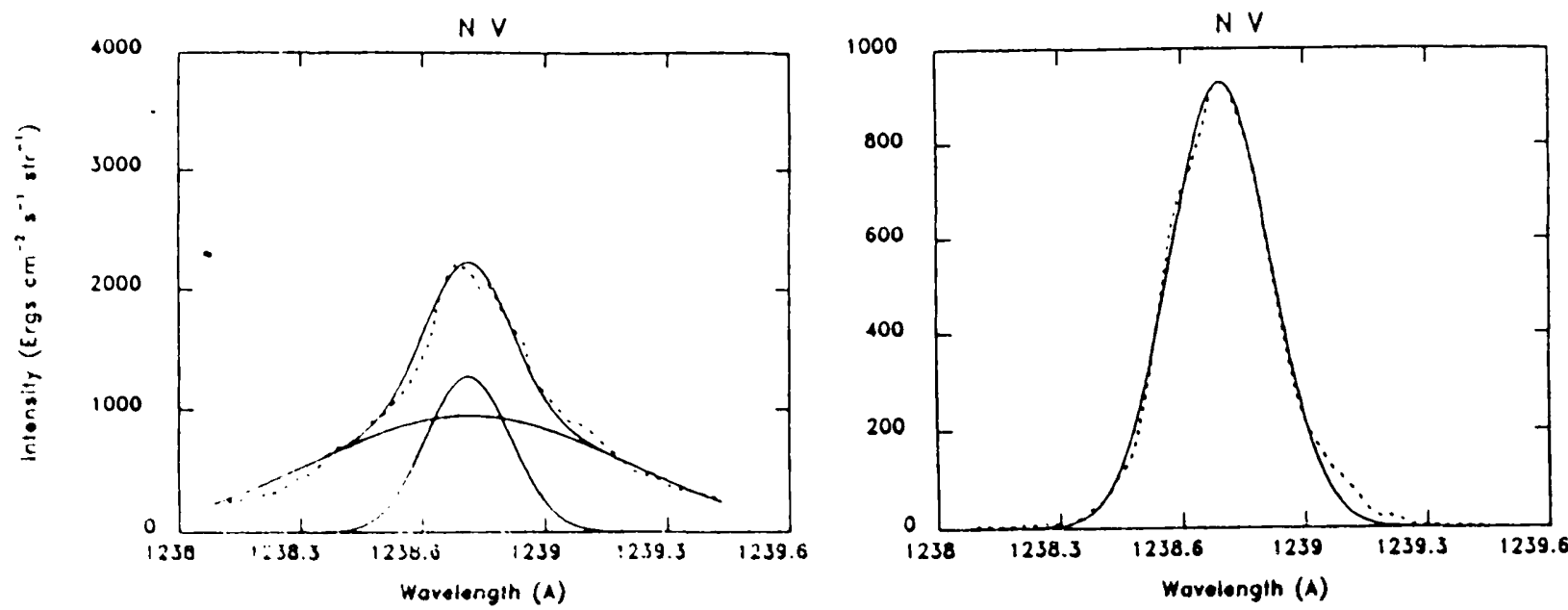

Fig. 3 

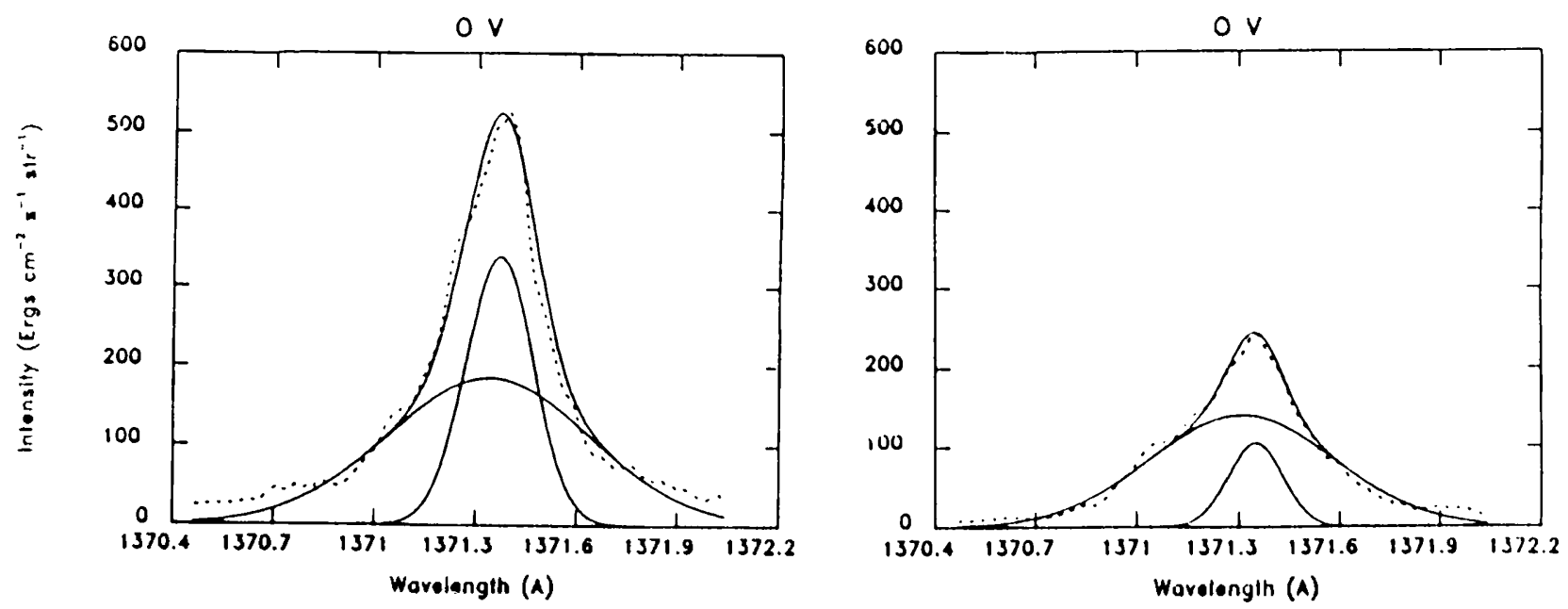

Fig. 4

typical of active region values. For the broad flare components the nonthermal turbulent velocity ranges from 90 to $140 \mathrm{~km} \mathrm{~s}^{-1}$ for profiles taken before and ciuring the impulsive hard $\mathrm{X}$-ray burst. During the decay phase the broadened component gradually disappeared, and the line profiles consisted of only the sharp active region components as shown by the Si IV and N V profiles at 2336 UT in the decay phase. In contrast to the transition region lines the chromospheric lines such às C I and O I lines did not show appreciable hroadening before and during the impulsive phase.

In the spectra taken before the onset of the impulsive HXR burst, at 2313 UT, the Fe XXI line was already present, indicating that the plasma heating had started in this earlier stage of the flare. Analysis of the Fe XXI line profiles shows that there were no blue-shifted components during the entire evolution of the flare. In addition, Fe XXI profiles were not broadened; all the profiles can be fitted by one gaussian with thermal Doppler width at $10^{7} \mathrm{~K}$. Figure 5 compares the Fe XXI profile taken before the impulsive phase (2314 UT) with that taken during the decay phase (2331 UT).
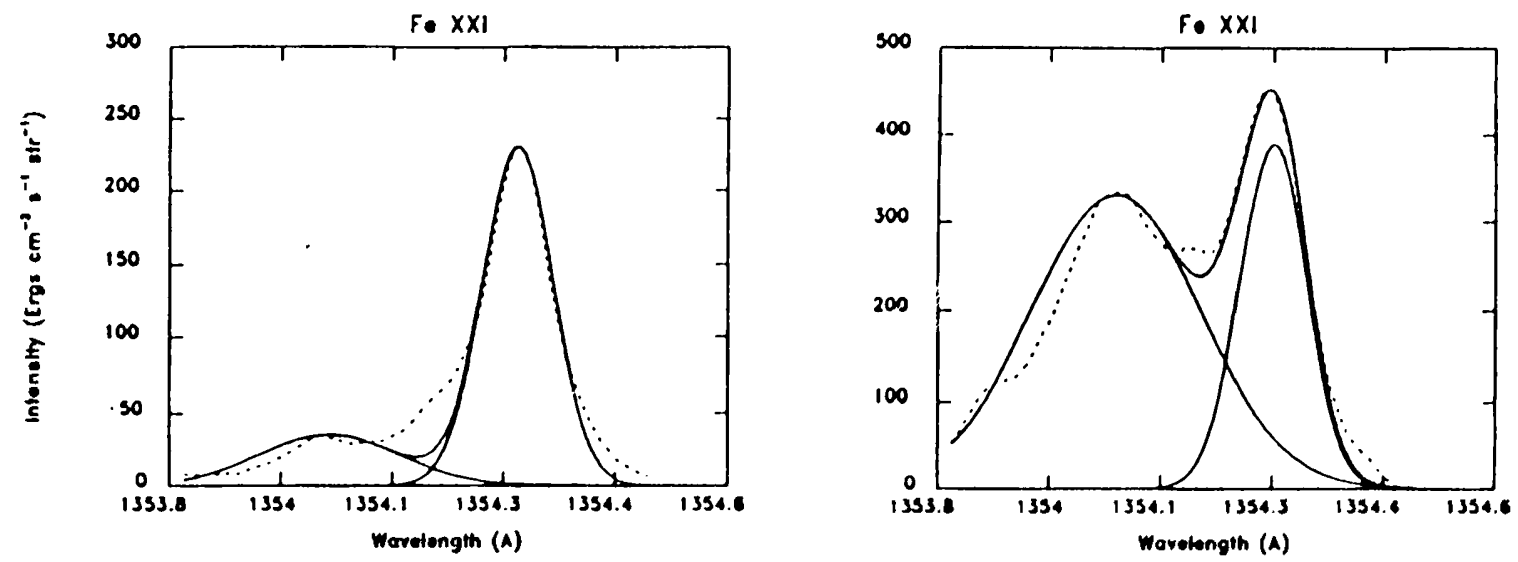

Fig. 5 
Density of the transition region plasmas is determined from the line ratios 0 IV (1399.8/1401.2) and Si III (1301.1/1296.7). The density determined from these ratios shows that the density was already high at about $10^{12} \mathrm{~cm}^{-3}$ in the beginning of the observation before and during the impulsive phase.

\section{SUMMARY AND DISCUSSION}

We found that: 1) Enhanced turbulence with nonthermal mass motion velocity of about $100 \mathrm{~km} \mathrm{~s}^{-1}$ was present in the transition region plasmas before the onset of the HXR burst. 2) The transition region plasmas were of high density before the onset of the HXR bursts. 3) Heating of the flare to $10^{7} \mathrm{~K}$ started before the impulsive phase. 4) There were no blue-shifted Fe XXI line profiles before, during, or after the impulsive phase.

These results show that enhanced turbulence and heating preceded the acceleration of energetic particles in the impulsive phase. It appears that the plasmas were first activated to create favorable conditions so that major instabilities as exhibited by the onset of the impulsive HXR burst could occur. The absence of blue-shifted components in the Fe XXI profiles shows that chromospheric evaporation is not an important energy and mass transport nechanism in this flare.

\section{REFERENCES}

Partoe, J.-D., Brueckner, G.E., Purcell, J.D., and Tousey, R. 1977, Appl. Optics, $16,879$.

Prueckner, G.E. 1976, Phil. Trans. Roy. Soc. London A, 281, 443.

Cheng, C.-C. 1977, AI.J., 213, 558.

Cheng, C.-C. 1978, Solar Phys., 56, 205.

Lere K.P., and Cook, J.W. 1979, AD.J., 229, 772.

l.oschek, G.A., Feldman, U., and Rosenberg, F.D. 1977, AD.J., 215, 329.

Viding, K.G., and Hiei, E. 1984, AD.J., 281, 426. 